\title{
IDENTIFICACIÓN, REFERENCIACIÓN Y ANÁLISIS DE LOS VECTORES ESTRATÉGICOS DEL PLAN ESTRATÉGICO DE CIENCIA, TECNOLOGÍA E INNOVACIÓN DEL TOLIMA
}

\author{
Alexis A. Aguilera Alvear \\ Centro de Productividad del Tolima \\ ctp@ctp.org.co
}

(Tipo de Artículo: Investigación. Recibido el 28/11/2012. Aprobado el 27/12/2012)

\begin{abstract}
RESUMEN
Como parte de las actividades metodológicas para la formulación del Plan Estratégico de Ciencia, Tecnología e Innovación del Tolima (PECTIT), se propone la realización de la referenciación internacional con el propósito de identificar los vectores estratégicos de Sistemas Regionales de Ciencia, Tecnología e Innovación (SRCTI) que han contribuido para un "buen u óptimo" desempeño del Sistema como parte del desarrollo socio-económico, productivo, ambiental y tecnológico de regiones extranjeras.
\end{abstract}

El ejercicio mencionado involucró a cinco regiones, ubicadas en Brasil, Chile, Italia, México y España, seleccionadas debido a sus buenas actuaciones sociales, ambientales y económicas, que se han basado en la ciencia, la tecnología y las estrategias de innovación.

La identificación de determinados vectores estratégicos apoyará RSTIS Tolima para priorizar estrategias y programas como componentes PECTIT, para ser ejecutado en el corto, mediano y largo plazo.

\section{Palabras clave}

Sistemas Regionales de Ciencia, Tecnología e Innovación; Vectores Estratégicos; Plan Estratégico de Ciencia, Tecnología e Innovación; Referenciación; Brechas; Tolima.

\section{IDENTIFICATION, BENCHMARKING AND ANALYSIS OF THE MAIN VECTORS OF THE STRATEGIC PLAN FOR SCIENCE, TECHNOLOGY AND INNOVATION OF TOLIMA-COLOMBIA}

\begin{abstract}
As part of the methodological activities for the development of the Strategic Plan for Science, Technology and Innovation of Tolima, Colombia, it is proposed the implementation of international benchmarking in order to identify the strategic vector of Regional Systems of Science, Technology and Innovation that have contributed to "good or excellent" performance of the system as part of socioeconomic, productive, environmental and technological development of foreign regions.
\end{abstract}

The above mentioned exercise involved five Regions, located in Brazil, Chile, Italy, Mexico and Spain, selected because of its good social, environmental and economic performances, which have been based on science, technology and innovation strategies.

The identification of selected strategic vectors will support Tolima RSTIS to prioritize strategies and programs like the components of the Strategic Plan for Science, Technology and Innovation of Tolima, in order to be executed in short, medium and long term.

\section{Keywords}

Regional Systems of Science, Technology and Innovation; Strategic Vectors; Strategic plan for Science, Technology and Innovation; Benchmarking; Gaps; Tolima.

\section{IDENTIFICATION, RÉFÉRENCEMENT ET ANALYSE DES VECTEURS PRINCIPAUX DU PLAN STRATÉGIQUE DE SCIENCE, TECHNOLOGIE ET INNOVATION DU TOLIMA-COLOMBIE}

\footnotetext{
Résumé

Comme une partie des activités méthodologiques pour la formulation du Plan Stratégique de Science, Technologie et Innovation du Tolima, Colombie, on propose la réalisation du référencement international avec le but d'identifier les vecteurs stratégiques du Systèmes Régionaux de Science, Technologie et Innovation (SRCTI) qui ont contribué à un "bon ou optimal " performance du Système comme partie du développement socio-économique, productif, environnemental et technologique des régions étrangères.
} 
Cet exercice est associé à cinq régions du Brésil, le Chili, l'Italie, le Mexique et l'Espagne qui ont été sélectionnés à cau se de leurs réalisations d'ordre social, environnemental et économique, qui se fondent sur la science, la technologie et des stratégies d'innovation.

L'identification de quelques vecteurs strategiques aidera à RSTIS pour prioriser des stratégies et programmes comme components du Plan Stratégique de Science, Technologie et Innovation pour les exécuter à court, moyen et long terme.

\section{Mots-clés}

Systèmes régionaux de Science, Technologie et Innovation, vecteurs strategiques, Plan Stratégique de Science, Technologie et Innovation, référencement, brèche, Tolima. 


\section{INTRODUCCIÓN}

La ley 1286 de 2009, Ley de Ciencia y Tecnología, establece entre sus propósitos y mandatos que el Departamento Administrativo de Ciencia, Tecnología e InnovaciónCOLCIENCIAS formule el Plan Nacional de Ciencia, Tecnología e Innovación teniendo en cuenta las metas y objetivos de los Planes Estratégicos Departamentales de Ciencia, Tecnología e Innovación (PEDCTI) y con ello cumpla con el objetivo establecido en dicha ley el cual pretende "fortalecer el desarrollo regional a través de políticas integrales de descentralización e internacionalización de las actividades científicas, tecnológicas y de innovación, de acuerdo con las dinámicas internacionales" [1].

De otra parte, la mencionada Ley establece en sus artículos 26, 27 y 28 que, le corresponde a las entidades territoriales incluir programas, proyectos y actividades de fomento de la ciencia, la tecnología y la innovación (CTel) en sus respectivos Planes de Desarrollo.

Colciencias como ente del Sistema Nacional de Ciencia, Tecnología e Innovación (SNCTI) se dio a la tarea de fomentar e impulsar la formulación de los PEDCTI con el propósito de dar cumplimiento a los mandatos de ley, integrar a los actores regionales en el SNCTI y lograr un modelo productivo social sustentado en la generación, uso y apropiación del conocimiento relacionado con las potencialidades y realidades de los territorios.

Inicialmente entre 2008 y 2009, Colciencias realizó un apoyo directo a través de convenios interinstitucionales a varios departamentos para la formulación de su PECTIT. Entre estos departamentos se encuentran: Antioquia, Risaralda, Bolívar, Valle del Cauca, Huila y Bogotá. En 2010 la convocatoria 539-2010 titulada "Conformar un Banco de Proyectos para Apoyar la Formulación de Planes Estratégicos Departamentales de Ciencia, Tecnología e Innovación" se lanzó para 23 departamentos, entre ellos el Tolima.

De esta manera, el Centro de Productividad del Tolima fue seleccionado como el ejecutor para la Formulación del PECTIT a partir de una metodología coherente y sistemática con los propósitos establecidos por Colciencias, entre los cuales se encuentra la realización de una referenciación internacional para la identificación de los ejes o vectores estratégicos, pilares de los programas y proyectos que se definan en el Plan.

En tal sentido, este artículo presenta los resultados de la referenciación internacional para el PECTIT.

\section{DESARROLLO}

La referenciación internacional para la identificación de los ejes o vectores estratégicos del PECTIT tomó como soporte metodológico las brechas tecnológicas y la vigilancia tecnológica.

En un sentido amplio el análisis de brechas busca la comparación con los mejores referentes con el ánimo de identificar, adaptar e implementar estrategias para mejorar los resultados en una organización [2]. Entre tanto, la vigilancia tecnológica es una metodología para la búsqueda, captación, procesamiento y análisis de información de carácter científico, tecnológico, político, normativo, económico, ambiental, entre otros para aportar elementos de juicio a la toma de decisiones en un campo, área, institución o región [3].
La aplicación de la metodología de brechas tecnológicas para desarrollar la referenciación internacional, tomó solo algunos elementos de ésta. En tal sentido, no se llegó hasta la comparación y medición de distancias entre los referentes y el Departamento del Tolima. La metodología de vigilancia tecnológica se aplicó para la búsqueda de información secundaria de los referentes seleccionados para los ejes estratégicos.

Para el caso del PECTIT, la referenciación parte de una serie de variables de la planificación en CTel de regiones internacionales, que den lugar a su análisis como modelo y con ello, estudiar la posibilidad de adaptar las prácticas de los referentes.

Por lo anterior, el desarrollo de la referenciación para el Plan parte de la metodología de brechas tecnológicas para realizar la definición de las variables o criterios a estudiar; la selección de los referentes; la construcción de diagnóstico del desempeño científico-tecnológico; y se apoya en la vigilancia tecnológica para la referenciación de cada variable o criterio; y la construcción de las conclusiones como insumos para el PECTIT. En síntesis, las actividades metodológicas son las siguientes.

\section{Tabla 1. Actividades metodológicas de la referenciación internacional}

\begin{tabular}{|c|c|c|}
\hline Metodología & Actividad & Producto \\
\hline \multirow{2}{*}{$\begin{array}{c}\text { Brechas } \\
\text { Tecnológicas }\end{array}$} & $\begin{array}{l}\text { Definición de los } \\
\text { criterios de la } \\
\text { referenciación } \\
\text { internacional }\end{array}$ & $\begin{array}{l}\text { Criterios para la } \\
\text { realización de la } \\
\text { referenciación }\end{array}$ \\
\hline & $\begin{array}{l}\text { Propuesta y } \\
\text { selección de } \\
\text { regiones }\end{array}$ & Regiones a estudiar \\
\hline \multirow{4}{*}{$\begin{array}{l}\text { Vigilancia } \\
\text { Tecnológica }\end{array}$} & $\begin{array}{l}\text { Identificación de la } \\
\text { estructura del } \\
\text { Sistema Regional } \\
\text { de Ciencia, } \\
\text { Tecnología e } \\
\text { Innovación de las } \\
\text { regiones referentes. }\end{array}$ & $\begin{array}{l}\text { Estructura, } \\
\text { políticas, actores, } \\
\text { programas del } \\
\text { Sistema Regional } \\
\text { de Ciencia, } \\
\text { Tecnología e } \\
\text { Innovación de } \\
\text { regiones referentes }\end{array}$ \\
\hline & $\begin{array}{l}\text { Referenciación de } \\
\text { las variables o } \\
\text { criterios (vectores y } \\
\text { estrategias de la } \\
\text { planificación de } \\
\text { ciencia, tecnología } \\
\text { e innovación) }\end{array}$ & $\begin{array}{l}\text { Referenciación de } \\
\text { las variables o } \\
\text { criterios para las } \\
\text { regiones } \\
\text { seleccionadas }\end{array}$ \\
\hline & Conclusiones & $\begin{array}{l}\text { Conclusiones para } \\
\text { el aporte al Plan } \\
\text { Estratégico de } \\
\text { Ciencia, Tecnología } \\
\text { e Innovación del } \\
\text { Tolima }\end{array}$ \\
\hline & $\begin{array}{l}\text { Socialización de los } \\
\text { resultados }\end{array}$ & $\begin{array}{l}\text { Presentación de } \\
\text { resultados }\end{array}$ \\
\hline
\end{tabular}

De igual manera, es importante mencionar que la referenciación internacional se condicionó a la disponibilidad de información secundaria, consultada en Internet, de cada uno de los referentes.

\subsection{Resultados}

En primer lugar, se hace referencia al concepto de Sistema Regional de Innovación (SRI) el cual no tiene una definición plenamente aceptada y siguiendo la exposición general que hacen Asheim y Gertler [4] se concibe como "la 
infraestructura institucional que apoya a la innovación en la estructura productiva de una región".

Por otra parte, un Sistema de Ciencia y Tecnología (SCyT) se compone de los actores que producen conocimiento en donde el rol principal lo tienen las universidades y los centros de investigación y desarrollo tecnológico.

En la integración de los dos conceptos anteriores surgen los Sistemas Regionales de Ciencia, Tecnología e Innovación (SRCTI) como modelo para el entendimiento de los arreglos para el fomento de la CTel en el ámbito local. Es precisamente, Cooke en 1992 quien habló por primera vez acerca del concepto integrador y señaló que los SRCTI están integrados por dos subsistemas: 1) Subsistema de generación de conocimiento o infraestructura de apoyo regional (laboratorios de investigación públicos y privados, universidades, agencias de transferencia tecnológica, organizaciones de formación continua) y 2) Subsistema de explotación de conocimiento o estructura de producción regional (empresas); lo cual permite integrar los conceptos de Sistema Nacional de Innovación (SNI) y SNCTI.

Así mismo, el Banco interamericano de desarrollo -BID- [5] identifica claramente cuatro subsistemas: 1) el Subsistema de generación de conocimiento en el cual se encuentran los actores como universidades, centros de investigación y demás agentes relacionados con la actividad académica y de investigación. 2) Subsistema de explotación del conocimiento conformado por las empresas. 3) Subsistema de apoyo a la investigación integrado por las instituciones de soporte a la infraestructura física, de maquinaria, de soporte tecnológico y de servicios avanzados. 4) Subsistema de financiación conformado por las instituciones del sector bancario, los agentes gubernamentales que disponen recursos para la investigación, las agencias de cooperación nacional e internacional, entre otros.

A partir de esta conceptualización se aborda la referenciación internacional como un proceso para conocer y comprender los ejes estratégicos concebidos como los soportes estructurales de los SRCTI de regiones pares al departamento del Tolima.

\subsection{Identificación y Selección de Referentes}

Para seleccionar las regiones referentes se estableció una serie de criterios, estos son:

- Referentes latinoamericanos y europeos.

- Apuestas productivas similares al Tolima (Agroindustrial - Agricultura - Textil - Turismo - Minería - Piscícola).

- Condiciones medioambientales similares (clima Tropical - sin tener en cuenta si es región costera).

- Política o plan de CTel.

Estos criterios tienen el propósito de identificar regiones extranjeras con condiciones ambientales y productivas con un grado de similaridad al Tolima y que cuenten con un direccionamiento claro y de largo plazo en materia de CTel. Así mismo, las regiones referentes son escogidas por contar con un desempeño socio-económico soportado en el desarrollo científico, tecnológico y de innovación. Bajo los cuatro (4) criterios mencionados, las regiones propuestas son:

- Pernambuco - Brasil [6]

- O’Higgins - Chile [7]

- Michoacán - México [8]

- Galicia - España [9]

- Emilia Romagna - Italia [10]
De igual manera, es importante mencionar que estas regiones cuentan con políticas y planes para fomento de la CTel y cada uno de estos define claramente los vectores del SRCTI y los programas asociados que permiten su desarrollo.

\subsection{Variables de comparación (vectores de los SRCTI)}

Con base en la selección de referentes de comparación, se procedió a realizar una revisión de las políticas, documentos de direccionamiento estratégicos o planes de CTel con el propósito de identificar y determinar los vectores estratégicos y sus respectivos sub-vectores de los SRCTI respectivos.

Es importante aclarar el significado que tiene los vectores estratégicos para efectos de la referenciación internacional. Por vector estratégico se entiende como los aspectos cruciales que actúan como obstaculizadores y facilitadores en el funcionamiento del SRCTI, por ende, son considerados como elementos relevantes para orientar el accionar de una política de la CTel.

Luego de la revisión de los Planes o Políticas de CTel de los referentes seleccionado, se propone el abordaje de las siguientes variables de comparación (vectores estratégicos) los cuales son comunes en todos los casos. Las variables de comparación son las siguientes.

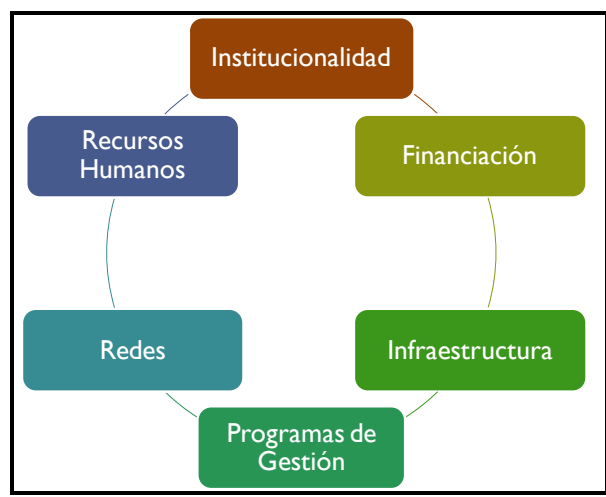

Fig. 1. Vectores estratégicos para referenciación internacional

Cada de una de estas variables de comparación (vectores) tiene, a su vez, una serie de sub-variables (sub-vectores). La revisión realizada a cada uno de los referentes permitió identificar los sub-vectores que dinamizan o desarrollan el eje respectivo. Estos son:

Tabla 2. Vector y sub-vectores de los SRCTI de regiones referentes

\begin{tabular}{|l|l|}
\hline \multicolumn{1}{|c|}{ Vector } & \multicolumn{1}{|c|}{ Sub-vectores } \\
\hline \multirow{4}{*}{ Institucionalidad } & $\checkmark$ Órgano rector \\
& $\checkmark$ Políticas de CTel \\
& $\checkmark$ Política regional conponente de CTel \\
& $\checkmark$ Actores \\
& $\checkmark$ Planes de CTel \\
\hline \multirow{3}{*}{ Recursos } & $\checkmark$ Maestría \\
Humanos & $\checkmark$ Movilidad \\
& $\checkmark$ Doctorados \\
\hline \multirow{2}{*}{ Financiación } & $\checkmark$ Jóvenes investigadores \\
\hline \multirow{2}{*}{ Redes } & $\checkmark$ Instrumentos de financiación \\
\hline Infraestructura de & $\checkmark$ Fondo regional de CTel \\
CTel & $\checkmark$ Cooperación nacional \\
& $\checkmark$ Sistema de información \\
\hline
\end{tabular}




\begin{tabular}{|l|l|}
\hline \multicolumn{1}{|c|}{ Vector } & Sub-vectores \\
\hline & desarrollo \\
\hline & $\checkmark$ Propiedad intelectual \\
& $\checkmark$ Emprendimiento \\
Programas de & $\checkmark$ Apropiación de CTel \\
gestión & $\checkmark$ Iniciación científica \\
& $\checkmark$ Apoyo a la innovación \\
& $\checkmark$ CTel aplicado a sectores \\
& estratégicos \\
\hline
\end{tabular}

\subsection{Comparación de referentes}

A continuación se presenta la comparación de los vectores y sub-vectores para cada una de las regiones referentes, incluyendo al Tolima.

Tabla 3. Referenciación de regiones para cada vector estratégico

\begin{tabular}{|c|c|c|c|c|c|c|c|}
\hline \multicolumn{2}{|c|}{$\frac{\text { g }}{\text { in }}$} & 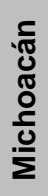 & 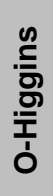 & $\frac{\text { 垔 }}{\frac{0}{0}}$ & 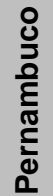 & 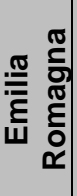 & $\frac{\text { 몽 }}{\frac{5}{0}}$ \\
\hline \multirow[b]{2}{*}{$\begin{array}{l}\frac{c}{0} \\
\frac{0}{0} \\
\frac{\pi}{0} \\
\frac{c}{0} \\
\frac{c}{1}\end{array}$} & $\begin{array}{l}\text { Fondo } \\
\text { Regional CTI }\end{array}$ & SI & NO & SI & NO & NO & $\mathrm{NO}^{* *}$ \\
\hline & $\begin{array}{l}\text { Instrumentos } \\
\text { de } \\
\text { financiación } \\
\text { (Convocatori } \\
\text { as) }\end{array}$ & SI & SI & SI & SI & SI & $\mathrm{SI}$ \\
\hline \multirow{5}{*}{ 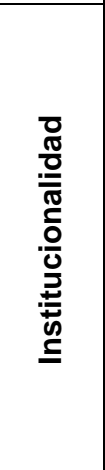 } & $\begin{array}{l}\text { Órgano } \\
\text { rector }\end{array}$ & SI & $\mathrm{SI}$ & SI & SI & SI & SI \\
\hline & Actores & SI & SI & SI & SI & SI & SI \\
\hline & $\begin{array}{l}\text { Política } \\
\text { Regional con } \\
\text { componente } \\
\text { CTI }\end{array}$ & SI & SI & NO & SI & NO & SI \\
\hline & $\begin{array}{l}\text { Política de } \\
\text { CTI (leyes, } \\
\text { decretos, } \\
\text { acuerdos) }\end{array}$ & SI & SI & SI & SI & SI & SI \\
\hline & Plan de CTI & SI & SI & SI & $\mathrm{NO}^{*}$ & SI & NO \\
\hline \multirow{6}{*}{ 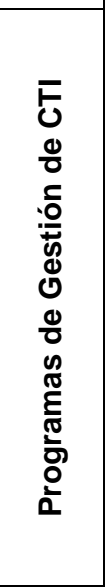 } & $\begin{array}{l}\text { Apropiación } \\
\text { CTI }\end{array}$ & SI & SI & SI & SI & SI & SI \\
\hline & $\begin{array}{l}\text { Sectores } \\
\text { Productivos o } \\
\text { Industriales }\end{array}$ & SI & SI & SI & $\mathrm{SI}$ & SI & $\mathrm{NO}^{* *}$ \\
\hline & $\begin{array}{l}\text { Apoyo a la } \\
\text { creación de } \\
\text { empresas de } \\
\text { base } \\
\text { tecnológica }\end{array}$ & SI & NO & SI & SI & SI & $\mathrm{NO}^{* *}$ \\
\hline & $\begin{array}{l}\text { Propiedad } \\
\text { Intelectual }\end{array}$ & NO & NO & NO & NO & SI & $\mathrm{NO}$ \\
\hline & $\begin{array}{l}\text { Iniciación } \\
\text { científica }\end{array}$ & SI & NO & NO & NO & NO & SI \\
\hline & $\begin{array}{l}\text { Apoyo a la } \\
\text { Innovación }\end{array}$ & NO & NO & SI & SI & SI & $\mathrm{NO}^{* *}$ \\
\hline \multirow{2}{*}{ 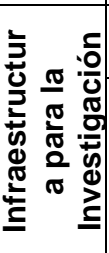 } & $\begin{array}{l}\text { Sistemas de } \\
\text { Información }\end{array}$ & SI & NO & NO & SI & SI & SI \\
\hline & $\begin{array}{l}\text { Dotación, } \\
\text { adquisición o } \\
\text { desarrollo de } \\
\text { infraestructur } \\
\text { a para la CTI }\end{array}$ & NO & NO & SI & SI & NO & NO \\
\hline
\end{tabular}

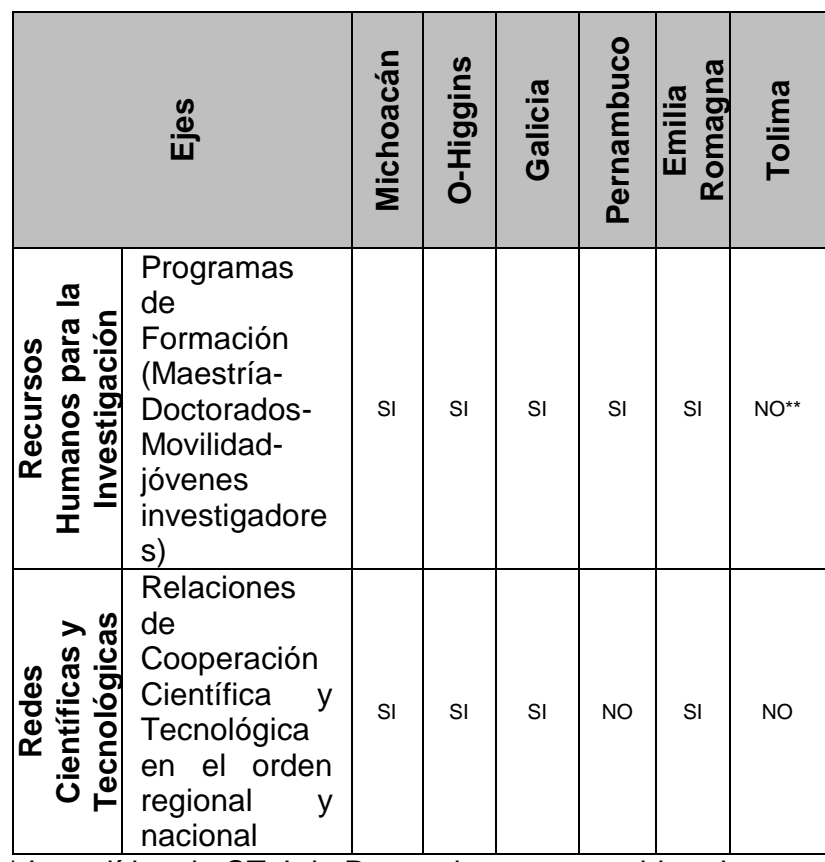

* La política de CTel de Pernambuco es considerada como el Plan de CTel.

** Programas promovidos por el Plan de Desarrollo del Tolima [11], pero que no se encuentran en ejecución u operación.

La revisión de los vectores y sub-vectores para las regiones referentes, permite establecer las siguientes conclusiones:

- Todos los vectores estratégicos son comunes en las regiones seleccionadas.

- Algunos sub-vectores son comunes para todas las regiones, entre ellos: instrumentos de financiación; reconocimiento oficial de actores de SRCTI; existencia de un órgano rector del SRCTI; programas de apropiación de CTel; programas de apoyo a sectores productivos y; programas de apoyo para la formación de recursos humanos en CTel.

- Los programas de iniciación científica (niños, jóvenes) solo se promueven en Michoacán (México) [8].

- Los programas de apoyo a la propiedad intelectual solo se promueven en Emilia Romagna (Italia) [10].

- Los fondos regionales de CTel se han constituidos en Michoacán y Galicia (España) [9], lo cual indica que no es una condición para desarrollar los SRCTI.

- Se puede afirmar que todas las regiones cuenta con planes de CTel. Aunque la matriz indica que Pernambuco no cuenta con planes de CTel, se considera que la política de CTel es el Plan de CTel, según lo manifestado en la propia política.

- Los sistemas de información son sub-vectores emergentes en los SRCTI analizados, toda vez que se han incorporado de manera reciente (últimos cuatro años) en regiones como Michoacán, Pernambuco y Emilia Romagna [6], [8], [10].

- Las políticas de desarrollo regional han posicionado a la CTel como componente de desarrollo. En tal sentido, se reconoce su existencia y ello posiciona a la CTel en la región, por medio de la creación de un órgano de dirección y coordinación, diseño e implementación de políticas y planes de CTel y apalancamiento de programas de gestión en la materia.

- La implementación de los programas de fomento a la CTel se realizan por medio de convocatorias en todas las regiones analizadas.

- Todas las regiones destinan recursos y diseñan programas específicos para incentivar la investigación 
aplicada y la innovación a mejorar los sectores productivos (o estratégicos) priorizados en las políticas regionales. Estos programas están orientados, principalmente, a las universidades y centros de investigación con el propósito de establecer alianzas con el sector productivo y empresarial.

- Los programas de apoyo a la innovación están orientados directamente a las empresas. Galicia, Pernambuco y Emilia Romagna cuentan con este tipo de programas.

\subsection{Vectores presentes en el Tolima}

Aunque en el Tolima se encuentran presenten los vectores de institucionalidad, financiación, programas de gestión de CTel, infraestructura y recursos humanos, los mismos solamente se encuentran planificados en la política del Tolima (Plan de Desarrollo del Tolima) [11], pero muy pocos con programas reales implementados. Solamente, el establecimiento de un órgano rector (institucionalidad), el Plan de Desarrollo del Tolima (Institucionalidad), la definición de los actores del SRCTI (institucionalidad), la política de CTel (institucionalidad) los programas de apropiación (Programas de gestión) y los sistemas de información (vector infraestructura) se encuentran en ejecución; los restantes solamente se encuentran enunciados en la política regional.

Lo anterior indica que el vector más desarrollado en el departamento del Tolima es el eje de institucionalidad, lo cual indica que el departamento cuenta con las bases estructurales de su sistema, es decir, con la política regional (Plan de Desarrollo del Tolima) [11] que incluye el componente de CTel, el órgano rector y una ordenanza vigente que permite definir los actores y reglas de juego del SRCTI.

Aunque en la Política Departamental se menciona que la CTel será el soporte para la competitividad del departamento, no se define explícitamente los sectores priorizados, ni los programas a implementar. Esto es una falencia de la planificación de CTel en el departamento.

El Departamento cuenta con un diferenciador de otros departamentos. El contar con un Observatorio de Ciencia y Tecnología departamental que realice el seguimiento y medición del desempeño científico y tecnológico es una ventaja que tiene el Tolima frente a otros departamentos, el cual permite dinamizar el sub-vector de sistemas de información.

El Fondo de CTel está establecido por ordenanza pero no se encuentra en ejecución, lo cual se constituye en una pérdida de capacidad y esfuerzo del departamento para el fomento de la CTel, toda vez que, los fondos son los dinamizadores de los vectores programas de gestión, infraestructura, recursos humanos y redes.

\section{CONCLUSIONES}

La referenciación internacional aporta elementos comunes y no comunes para estructurar los planes de CTel. En este sentido, los ejes recurrentes en los sistemas regionales de innovación analizados son:

- Institucionalidad

- Recursos Humanos

- Programas de gestión para capacidades científicas y tecnológicas

- Financiación

Los vectores mencionados anteriormente son los cuatro ejes que la literatura y la teoría sobre SRCTI mencionan. Ello significa que las regiones analizadas han tomado como soporte estructural las propuestas teóricas y las han implementado por medio de los sub-vectores, dando resultados positivos.

Los vectores "infraestructura" y "redes científicas y tecnológicas" pueden ser consideradas como emergentes, dado que no todas las regiones las consideran como vectores estructurales del Sistema o de los componentes de los Planes.

Los anteriores no se encuentran en las teorías de SRCTI como vectores o ejes. Se manifiestan como funciones transversales para que un sistema funcione correctamente. En tal sentido, la importancia que destacan estos en las regiones analizadas como parte central del SRCTI demuestra que la infraestructura y la información son necesarias para implementar programas de CTel.

Es importante aclarar que en algunos planes regionales de CTel (Brasil y México) consideran el vector de infraestructura como parte de los programas para el desarrollo de capacidades.

Uno de los aspectos fundamentales para el buen desempeño de los SRCTI analizados es la financiación para la promoción de los programas. Algunas regiones han implementado fondos regionales para diferentes programas, otras regiones fondos sectoriales o por programas.

Un aspecto fundamental para el correcto desempeño de los SRCTI de los casos revisados se constituye en el liderazgo claro por parte de una única entidad, que orienta las estrategias y programas de CTel, evitando así la duplicidad de esfuerzos y lineamientos.

Así mismo, otra condición estratégica es la participación e involucramiento directo de los agentes de referencia del SRCTI, que para todos los casos involucra al Estado, la Academia y la Empresa, teniendo a la sociedad como beneficiaria.

El involucramiento de los actores del SRCTI se da desde el aporte financiero, el diseño de políticas y programas, la ejecución de los mismos y el seguimiento.

La priorización de sectores permite focalizar los programas de CTel y el desarrollo de capacidades para potenciar la competitividad regional basada en conocimiento.

Un hecho de suma importancia lo presenta Emilia Romagna [10] con la implementación del Programa de Propiedad Intelectual, toda vez que es la única región con tal programa, lo cual demuestra conciencia e importancia de la generación y protección del conocimiento para su aplicación a la solución de necesidades.

Por último, uno de los aspectos que llama la atención se centra en los programas de apoyo al emprendimiento de base tecnológica. Ello demuestra una clara tendencia a la transformación de la estructura productiva para incorporar el conocimiento en como parte de las estrategias de productividad y competitividad.

Con base en la revisión y análisis de la referenciación internacional, las principales recomendaciones para el SRCTI del Tolima pueden resumirse en los siguientes puntos:

1. EI SRCTI del Tolima deberá determinar los sectores socio-económicos sobre los cuales apuntarán los programas y proyectos (Caso Chile - Brasil - España), basado precisamente sobre los documentos regionales de competitividad como lo son la Agenda Interna de 
Productividad y Competitividad, el Plan Regional de Competitividad, la Agenda Prospectiva de Ciencia y Tecnología, entre otros.

2. Determinar claramente las funciones de los actores involucrados en el SRCTI (Caso España). Para el departamento del Tolima es sumamente importante establecer roles, según los roles de los actores, según Colciencias (2008) [12] tales como financiación, información, científico, tecnológico y gobernanza y de esta manera apoyar al órgano rector (Consejo Departamental de Ciencia, Tecnología e Innovación del Tolima) para movilizar y realizar el seguimiento de los programas de fomento.

3. Revisar la constitución y funcionamiento (fuentes de financiación) de los fondos regionales para CTel a partir de los modelos implementados en departamentos como el Huila, Risaralda, Bolívar y Antioquia. Igualmente, otro de los referentes que se sugiere revisar es el Fondo de CTel de Colombia, el Fondo Nacional de Financiamiento la Ciencia, la Tecnología y la Innovación "Fondo Francisco José de Caldas". Estos modelos permitirán identificar las fuentes de financiación, los esquemas de manejo de recursos y los mecanismos e instrumentos para la financiación de programas y proyectos del Plan de CTel del Tolima.

4. Asegurar que la estructura del SRCTI del Tolima cuente con los cuatro vectores centrales (Institucionalidad Financiación - Recursos Humanos - Programas de Gestión), de tal manera que los programas implementados en la actualidad continúen su operación. Adicionalmente, estudiar la posibilidad de implementar otros programas conexos de cada vector identificados en la referenciación internacional que permitan dinamizar el SRCTI.

5. Específicamente, para el vector de infraestructura, el Tolima ha avanzado positivamente en la gestión de la información, a través del Observatorio de Ciencia, Tecnología e Innovación. Sin embargo, aún el Departamento depende de los indicadores de CTel nacionales que genera el Observatorio Colombiano de Ciencia y Tecnología (OCyT), entre ellos datos sobre inversión, capacidades (grupos, investigadores), producción científica, entre otros, y aún no se generan indicadores específicos para los programas de fomento implementados en la región, por ejemplo, Programa Ondas Tolima. En tal sentido, la recomendación se centra en la generación de una batería de indicadores a la medida de los programas de CTel que se encuentren operando en el departamento, incluyendo la medición de los programas y proyectos establecidos en el PECTIT.

6. Por otra parte, es importante que el Departamento establezca programas de fortalecimiento de la infraestructura para la CTel ligados a los centros, laboratorios y grupos de investigación según los sectores priorizados por la Comisión Regional de Competitividad del Tolima y los que se prioricen en el PECTIT para focalizar los programas científicos y tecnológicos de apoyo a la productividad y competitividad. En tal sentido, es pertinente seguir el modelo Brasil (Programa de fortalecimiento de la infraestructura para la investigación en centros de investigación, laboratorios, universidades y empresas) el cual opera por medio de convocatorias y solamente financia aquella infraestructura para la generación de conocimiento que aplique para proyectos de investigación e innovación para sectores productivos o industriales y en el cual participen los centros, universidades, laboratorios y grupos de investigación.

7. Es muy importante que el Departamento considere la gestión de la propiedad intelectual como mecanismo para el fomento de la investigación e innovación, principalmente en el sector productivo, empresarial e industrial de la región.

8. En cuanto al vector de Redes Científicas y Tecnológicas, el Tolima cuenta con una gran oportunidad para hacer parte de las redes nacionales e internacionales en diferentes áreas de conocimiento. En tal sentido, la incorporación de las redes de conocimiento debe tener como lineamiento aquellos temas o sectores priorizados en el PECTIT. Implementar este vector permitirá que Departamento pueda contar con infraestructura, capital humano, financiamiento de proyectos por medio de alianzas estratégicas.

\section{REFERENCIAS}

[1] Congreso de la República. Ley 1286 de 2009 "Por la cual se modifica la Ley 29 de 1990, se transforma a Colciencias en Departamento Administrativo, se fortalece el Sistema Nacional de Ciencia, Tecnología e Innovación en Colombia y se dictan otras disposiciones". Artículo 2 "Objetivos Específicos". Diario Oficial $\mathrm{N}^{\circ}$ 47.241. Bogotá. Online [En. 2009].

[2] J. Medina, A. Aguilera, C. Franco \& L. Landines, Modelo Prospectiva y Vigilancia Tecnológica del Sena para la Respuesta Institucional de Formación - Manual Serie Guías Metodológicas, Cali: Instituto de Prospectiva, Innovación y Gestión del Conocimiento, Universidad del Valle. 2010.

[3] F. Palop \& J. M. Vicente, Vigilancia tecnológica e Inteligencia competitiva. Su potencial para la empresa Española, Madrid: COTEC. 1999.

[4] B. T. Asheim \& M. S. Gertler, "The geography of innovation: regional innovation systems," in Fagerberg, J., Mowery, D., Nelson, R. (Eds.), The Oxford Handbook of Innovation. Oxford University Press, Oxford, pp. 291317. 2005.

[5] J. J. Llisterri \& C. Pietrobelli. Los Sistemas Regionales de Innovación en América Latina. Washington: Banco Interamericano de Desarrollo BID, 2011.

[6] Gobierno de Pernambuco. Decreto No 30.369 del 19 de abril de 2007. Reglamento de la Secretaria de Ciencia, Tecnología y Medio Ambiente y dicta otras providencias. Recife. Online [Feb. 2012].

[7] Instituto de Desarrollo Local y Regional, IDER, Universidad de La Frontera. Política de Ciencia, Tecnología e Innovación. Región del Libertador Bernardo O'Higgins. Online [Ago. 2010].

[8] Consejo Estatal de Ciencia y Tecnología de Michoacán, "Programa Estatal de Ciencia y Tecnología de Michoacán. Morelia". Online [Mar. 2006].

[9] Junta de Galicia. Plan Gallego de Investigación, Innovación y Crecimiento 2011-2015. Galicia. Online [Jul. 2011].

[10] R. E. Romagna. "Programa Regional de Investigación Industrial, Innovación y Transferencia Tecnológica. 2012-2015". Italia. Online [En. 2011].

[11] Gobernación del Tolima. Plan de Desarrollo Departamental 2012-1015. Online [Feb. 2012].

[12] Instituto Colombiano para el Desarrollo de la Ciencia y la Tecnología Francisco José de Caldas COLCIENCIAS. Sistema Nacional de Ciencia, Tecnología e Innovación. Presentación Institucional. Oficina de Planeación: Bogotá. 2008. 